SHEP 01-06

\title{
CONVERGENCE OF DERIVATIVE EXPANSIONS IN SCALAR FIELD THEORY
}

\author{
TIM R. MORRIS ${ }^{1}$ AND JOHN F. TIGHE ${ }^{2}$ \\ Department of Physics, University of Southampton, \\ Highfield, Southampton SO17 1BJ, UK \\ ${ }^{1}$ E-mail: trmorris@hep.phys.soton.ac.uk \\ ${ }^{2}$ E-mail: jft@hep.phys.soton.ac.uk
}

\begin{abstract}
The convergence of the derivative expansion of the exact renormalisation group is investigated via the computation of the $\beta$ function of massless scalar $\lambda \varphi^{4}$ theory. The derivative expansion of the Polchinski flow equation converges at one loop for certain fast falling smooth cutoffs. Convergence of the derivative expansion of the Legendre flow equation is trivial at one loop, but also can occur at two loops and in particular converges for an exponential cutoff.
\end{abstract}

\section{Introduction}

The derivative expansion of the effective action within the context of the exact renormalisation group has been shown to provide an accurate non-perturbative approximation method for scalar quantum field theory 1 . While this statement rests on empirical fact, it is a challenging task to prove the applicability of the derivative expansion non-perturbatively and in all generality since it is not a controlled expansion in a small parameter. Rather, it results in a numerical series since the approximation lies with neglecting higher powers of $p / \Lambda$ (where $\Lambda$ is the effective cutoff and $p$ some typical momentum) and the flow equations require the contributing typical momentum to be of order $\Lambda$. Thus it is a non-trivial question to ask whether such an expansion converges and, if so, whether it converges to the correct answer. Here we address this question within perturbation theory.

\section{Wilson/Polchinski Flow Equation}

We define modified propagators $\Delta_{U V}=C_{U V}\left(q^{2} / \Lambda^{2}\right)$ and $\Delta_{I R}=C_{I R}\left(q^{2} / \Lambda^{2}\right)$, where $C_{U V}\left(C_{I R}\right)$ is an as yet unspecified function acting as an UV (IR) cutoff with the properties $C_{U V}(0)=1$ and $C_{U V} \rightarrow 0$ (sufficiently fast) as $q \rightarrow \infty$, with $C_{I R} \equiv 1-C_{U V}$. We write Polchinski's versiont of Wilson's flow equation for the individual vertices as

$$
\begin{aligned}
\frac{\partial}{\partial \Lambda} S\left(\mathbf{p}_{1}, \cdots, \mathbf{p}_{n} ; \Lambda\right)= & \sum_{\left\{I_{1}, I_{2}\right\}} S\left(-\mathbf{P}_{1}, I_{1} ; \Lambda\right) \frac{d}{d \Lambda} \Delta_{U V}\left(P_{1}\right) S\left(\mathbf{P}_{1}, I_{2} ; \Lambda\right) \\
& -\frac{1}{2} \int \frac{d^{4} q}{(2 \pi)^{4}} \frac{d}{d \Lambda} \Delta_{U V}(q) S\left(\mathbf{q},-\mathbf{q}, \mathbf{p}_{1}, \cdots, \mathbf{p}_{n} ; \Lambda\right),
\end{aligned}
$$

where $I_{1}$ and $I_{2}$ are disjoint subsets of external momenta such that $I_{1} \cap I_{2}=\emptyset$ and $I_{1} \cup I_{2}=\left\{\mathbf{p}_{1}, \cdots, \mathbf{p}_{n}\right\}$. The sum over $\left\{I_{1}, I_{2}\right\}$ utilises the Bose symmetry so pairs are counted only once i.e. $\left\{I_{1}, I_{2}\right\}=\left\{I_{2}, I_{1}\right\}$. The momentum $\mathbf{P}_{1}$ is defined to be $\mathbf{P}_{1}=\sum_{\mathbf{p}_{i} \in I_{1}} \mathbf{p}_{i}$. 


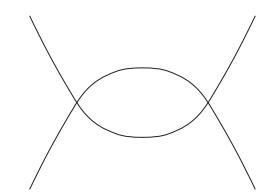

Figure 1. Feynman diagram contributing to four-point function at one loop
With the renormalisation condition $S(0,0,0,0 ; \Lambda)=\lambda$ imposed and the $\beta$ function defined as $\beta(\Lambda) \equiv \Lambda \frac{\partial}{\partial \Lambda} \lambda$, the only contribution to the $\beta$ function at one loop takes the form of figure 11. Within the flow equation, this contribution arises from the tree-level six-point function with two of its legs tied together.

The $\beta$ function at one loop is found to be

$$
\begin{aligned}
\beta & =3 \lambda^{2} \Lambda \int \frac{d^{4} q}{(2 \pi)^{4}} \frac{d}{d \Lambda} \Delta_{U V}\left(q^{2} / \Lambda^{2}\right)\left[\int_{\Lambda}^{\infty} d \Lambda_{1} \frac{d}{d \Lambda_{1}} \Delta_{U V}\left(q^{2} / \Lambda_{1}^{2}\right)\right] \\
& =\frac{6 \lambda^{2}}{(4 \pi)^{2}} \sum_{n=1}^{\infty} \frac{C_{U V}^{(n)}(0)}{n !} \int_{0}^{\infty} d x x^{n} C_{U V}^{\prime}(x),
\end{aligned}
$$

where we have taken the opportunity to perform a derivative expansion. The derivative expansion corresponds to an expansion in the momentum dependent part of the six-point function which relates to the term in square brackets in the first line of (2). This expression is evidently dependent on the exact form of the cutoff function. Since the sharp cutoff should not be considered within the context of the Wilson/Polchinski flow equation, we shall restrict ourselves to smooth profiles. With an exponential cutoff of the form $C_{U V}\left(q^{2} / \Lambda^{2}\right)=e^{-q^{2} / \Lambda^{2}}$, problems arise:

$$
\beta=\frac{6 \lambda^{2}}{(4 \pi)^{2}} \sum_{n=1}^{\infty}(-1)^{n+1}
$$

Clearly this fails to converge to the correct value of $\beta=\frac{3 \lambda^{2}}{(4 \pi)^{2}}$, and indeed exponentials of any power (i.e. $C_{U V}(x)=e^{-x^{m}}$ ) suffer from the same affliction. However there are UV cutoffs that can provide the desired convergence. If we consider the much faster falling $C_{U V}(x)=\exp \left(1-e^{x}\right)$, we obtain the converging series

$$
\beta=\frac{3 \lambda^{2}}{(4 \pi)^{2}}\{1.193+0-0.194-0.060+0.032+\cdots\} .
$$

\section{Legendre flow equation at one loop}

Irrespective of the exact form of the cutoff, the only contribution to the flow of the one-loop four-point $1 \mathrm{PI}$ is

$$
\begin{aligned}
& \frac{\partial}{\partial \Lambda} \Gamma\left(\mathbf{p}_{1}, \mathbf{p}_{2}, \mathbf{p}_{3}, \mathbf{p}_{4} ; \Lambda\right)=\int \frac{d^{4} q}{(2 \pi)^{4}} K_{\Lambda}(q) \\
& \quad \times \sum_{\left\{I_{1}, I_{2}\right\}} \Gamma\left(\mathbf{q},-\mathbf{q}-\mathbf{P}_{1}, I_{1} ; \Lambda\right) \Delta_{I R}\left(\left|\mathbf{q}+\mathbf{P}_{1}\right|\right) \Gamma\left(\mathbf{q}-\mathbf{P}_{2},-\mathbf{q}, I_{2} ; \Lambda\right),
\end{aligned}
$$

where the notation is the same as used in (11). Imposing the renormalisation condition $\Gamma(0,0,0,0 ; \Lambda)=\lambda$ and substituting the classical vertex $\lambda$ for the four-point 
functions on the right hand side of (5), we find that

$$
\beta=3 \lambda^{2} \Lambda \int \frac{d^{4} q}{(2 \pi)^{4}}\left(\frac{d}{d \Lambda} \Delta_{U V}\left(q^{2} / \Lambda^{2}\right)\right) \Delta_{I R}\left(q^{2} / \Lambda^{2}\right)=\frac{3 \lambda^{2}}{(4 \pi)^{2}} .
$$

Note that the result is independent of cutoff function as expected, and exact irrespective of the derivative expansion because the classical four-point vertex carries no external momentum dependence.

\section{Sharp cutoff at two loops}

Various forms of the sharp cutoff version in vertices can be found in the literature. 6.3 By the process of iteration, the usual Feynman diagrams can be constructed but with restrictions on the allowed values of momentum for internal propagators.

To ensure that only renormalised quantities are used, we split the four point function into its momentum free $[\lambda(\Lambda)]$ and momentum dependent parts:

$$
\Gamma\left(\mathbf{p}_{1}, \mathbf{p}_{2}, \mathbf{p}_{3}, \mathbf{p}_{4} ; \Lambda\right)=\lambda(\Lambda)+\gamma\left(\mathbf{p}_{1}, \mathbf{p}_{2}, \mathbf{p}_{3}, \mathbf{p}_{4} ; \Lambda\right),
$$

with $\gamma(0,0,0,0 ; \Lambda)=0$. Momentum expanding the one-loop result, we find

$$
\begin{aligned}
& \gamma\left(\mathbf{p}_{1}, \mathbf{p}_{2}, \mathbf{p}_{3}, \mathbf{p}_{4} ; \Lambda\right) \\
& =-\lambda^{2} \int_{\Lambda}^{\infty} d \Lambda_{1} \int \frac{d^{4} q}{(2 \pi)^{4}} \frac{\delta\left(q-\Lambda_{1}\right)}{q^{2}} \sum_{i=2}^{4}\left\{\frac{\theta\left(\left|\mathbf{q}+\mathcal{P}_{i}\right|-\Lambda_{1}\right)}{\left(\mathbf{q}+\mathcal{P}_{i}\right)^{2}}-\frac{\theta\left(q-\Lambda_{1}\right)}{q^{2}}\right\} \\
& =+\frac{\lambda^{2}}{4 \pi^{3}} \sum_{i=2}^{4}\left\{\frac{1}{6} \frac{\mathcal{P}_{i}}{\Lambda}+\frac{1}{720}\left(\frac{\mathcal{P}_{i}}{\Lambda}\right)^{3}+\frac{3}{44800}\left(\frac{\mathcal{P}_{i}}{\Lambda}\right)^{5}+\cdots\right\},
\end{aligned}
$$

It is $\gamma\left(\mathbf{p}_{1}, \mathbf{p}_{2}, \mathbf{p}_{3}, \mathbf{p}_{4} ; \Lambda\right)$ that is iterated through the flow equation to obtain the running of the coupling to second loop order.

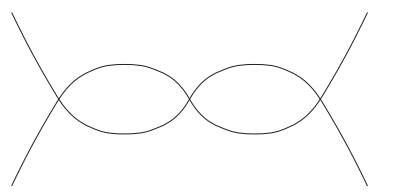

(a)

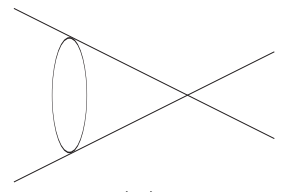

(b)

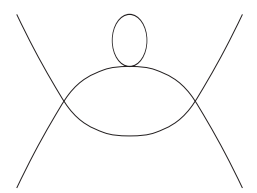

(c)

Figure 2. Feynman diagrams contributing to the four-point function at two loops.

The three possible topologies of two-loop four-point 1PI diagrams are displayed in figure 2. Topology (a) does not provide a contribution to the $\beta$ function; in terms of renormalised quantities (a) is already incorporated in the one-loop running $\lambda(\Lambda)$. There are two contributions of the form of (c), one arising from the one-loop self energy being inserted into the one-loop four-point function and the other from the one-loop six-point 1PI diagram with two legs at the same vertex joined together. It can be shown that for all types of cutoff function, these two contributions cancel one another. Hence the only contributions we need consider are those of topology 
(b). The first comes from the iteration of the renormalised one-loop four-point function and its contribution to the $\beta$ function is

$$
\begin{aligned}
& -6 \lambda \Lambda \int \frac{d^{4} q}{(2 \pi)^{4}} \frac{\delta(q-\Lambda)}{q^{2}} \frac{\theta(q-\Lambda)}{q^{2}} \gamma(\mathbf{q},-\mathbf{q}, 0,0 ; \Lambda) \\
& =\frac{\lambda^{3}}{(4 \pi)^{4}} \frac{1}{\pi}\left(8+\frac{1}{15}+\frac{9}{2800}+\cdots\right) .
\end{aligned}
$$

The next two parts arise from the one-loop six-point 1PI diagram with two legs from different vertices joined up. The first contributes

$$
\begin{aligned}
& -6 \lambda^{3} \int \frac{d^{4} q}{(2 \pi)^{4}} \frac{\delta(q-\Lambda)}{q^{2}} \int_{\Lambda}^{\infty} d \Lambda_{1} \int \frac{d^{4} p}{(2 \pi)^{4}} \frac{\delta\left(p-\Lambda_{1}\right)}{p^{2}} \frac{\theta^{2}\left(|\mathbf{p}+\mathbf{q}|-\Lambda_{1}\right)}{|\mathbf{p}+\mathbf{q}|^{4}} \\
& =-12 \frac{\lambda^{3}}{(4 \pi)^{4}} \frac{1}{\Lambda} \frac{1}{\pi}\left(\frac{\pi}{2}-\frac{10}{9}+\frac{\pi}{4}-\frac{63}{100}+\frac{\pi}{6}+\cdots\right),
\end{aligned}
$$

while the other is

$$
\begin{aligned}
& -12 \lambda^{3} \int \frac{d^{4} q}{(2 \pi)^{4}} \frac{\delta(q-\Lambda)}{q^{2}} \int_{\Lambda}^{\infty} d \Lambda_{1} \int \frac{d^{4} p}{(2 \pi)^{4}} \frac{\delta\left(p-\Lambda_{1}\right)}{p^{2}} \frac{\theta\left(|\mathbf{p}+\mathbf{q}|-\Lambda_{1}\right) \theta\left(p-\Lambda_{1}\right)}{p^{2}|\mathbf{p}+\mathbf{q}|^{2}} \\
& =-12 \frac{\lambda^{3}}{(4 \pi)^{4}} \frac{1}{\Lambda} \frac{1}{\pi}\left(\frac{\pi}{2}-\frac{2}{9}-\frac{1}{300}-\frac{3}{15680}+\cdots\right) .
\end{aligned}
$$

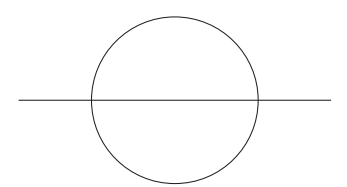

Figure 3. Feynman diagram contributing to wave function renormalization at two loops.
While both of these series converge, the first only does so very slowly and indeed for a $O\left(\partial^{2}\right)$ operator (or higher) this diagram would diverge.

The final contribution to the $\beta$ function at two-loop order takes account of the wavefunction renormalisation due to figure 3 which appears through $\left.\Sigma(k ; \Lambda)\right|_{O\left(k^{2}\right)}=$ $[Z(\Lambda)-1] k^{2}$.

From this we find

$$
\begin{aligned}
& k^{2} \frac{\partial}{\partial \Lambda} Z(\Lambda) \\
& \quad=\left.\lambda^{2} \int \frac{d^{4} q}{(2 \pi)^{4}} \frac{\delta(q-\Lambda)}{q^{2}} \int_{\Lambda}^{\infty} d \Lambda_{1} \int \frac{d^{4} p}{(2 \pi)^{4}} \frac{\delta\left(p-\Lambda_{1}\right)}{p^{2}} \frac{\theta\left(|\mathbf{p}+\mathbf{q}+\mathbf{k}|-\Lambda_{1}\right)}{|\mathbf{p}+\mathbf{q}+\mathbf{k}|^{2}}\right|_{O\left(k^{2}\right)} \\
& =-\frac{\lambda^{2} k^{2}}{(4 \pi)^{4}} \frac{1}{\Lambda} \frac{1}{\pi}\left(\frac{1}{2}+\frac{1}{48}+\frac{3}{1280}+\cdots\right)
\end{aligned}
$$

Altogether, these contributions converge towards the correct two-loop answer for the properly normalised $\beta$ function

$$
\beta(\Lambda)=\Lambda \frac{\partial}{\partial \Lambda}\left(\frac{\lambda(\Lambda)}{Z^{2}}\right)=3 \frac{\lambda^{2}}{(4 \pi)^{2}}-\frac{17}{3} \frac{\lambda^{3}}{(4 \pi)^{4}} .
$$




\section{Smooth cutoffs}

When a smooth cutoff is utilised, the fegendre flow equation takes on a slightly different form to its sharp counterpart.6.3

If we consider a power law cutoff $C_{U V}\left(q^{2} / \Lambda^{2}\right)=\frac{1}{1+(q / \Lambda)^{2 \kappa+2}}$ (with $\kappa$ a nonnegative integer) the derivative expansion fails at two loops. For instance with the iteration of the one-loop four-point function, we have the following contribution to the two-loop $\beta$ function:

$$
\begin{aligned}
& \sim \frac{\lambda^{3}}{\Lambda^{2 \kappa+3}} \int d^{4} q \frac{q^{4 \kappa}}{\left[1+(q / \Lambda)^{2 \kappa+2}\right]^{3}} \int_{\Lambda}^{\infty} \frac{d \Lambda_{1}}{\Lambda_{1}^{2 \kappa+3}} \int d^{4} p \times \\
& \quad \times \frac{p^{2 \kappa}}{\left[1+\left(p / \Lambda_{1}\right)^{2 \kappa+2}\right]^{2}}\left[1-\frac{1}{1+\left(|\mathbf{q}+\mathbf{p}| / \Lambda_{1}\right)^{2 \kappa+2}}\right] \frac{1}{|\mathbf{q}+\mathbf{p}|^{2}} .
\end{aligned}
$$

With a derivative expansion the inner integral is expanded in terms of $q$, but when the power $q^{2 m}$ is such that $m \geq \kappa+1$, the outer integral fails to converge. Hence the coefficients of the derivative expansion are themselves infinite.

However, the situation is much better when an exponential cutoff ${ }^{a}$ of the form $C_{U V}\left(q^{2} / \Lambda^{2}\right)=e^{-q^{2} / \Lambda^{2}}$ is used. The renormalised one-loop four-point function is

$$
\gamma\left(\mathbf{p}_{1}, \mathbf{p}_{2}, \mathbf{p}_{3}, \mathbf{p}_{4} ; \Lambda\right)=-\frac{\lambda^{2}}{2(4 \pi)^{2}} \sum_{i=2}^{4} \sum_{n=1}^{\infty} \frac{(-1)^{n}}{(n+1) ! n}\left(\frac{\mathcal{P}_{i}{ }^{2}}{2 \Lambda_{1}{ }^{2}}\right)^{n} .
$$

When this is iterated through the flow equation, its contribution to the $\beta$ function is 9

$$
-12 \frac{\lambda^{3}}{(4 \pi)^{4}} \frac{1}{\Lambda} \sum_{n=1}^{\infty} \frac{(-1)^{n}}{n(n+1)}\left(\frac{1}{2^{n}}-\frac{1}{2^{2 n+1}}\right)
$$

which can be shown to sum exactly to $\frac{6 \lambda^{3}}{(4 \pi)^{4}} \frac{1}{\Lambda}[6 \ln 3+4 \ln 2-5 \ln 5-1]$. The analogue of (11) is

$$
12 \frac{\lambda^{3}}{(4 \pi)^{4}} \frac{1}{\Lambda}\left(\ln \frac{4}{3}+\sum_{n=2}^{\infty}(-1)^{n}\left[\ln \frac{4}{3}-\frac{1}{n} \sum_{s=2}^{n}\left(\begin{array}{l}
n \\
s
\end{array}\right) \frac{(-1)^{s}}{s-1}\left\{1-\frac{1}{2^{s-2}}+\frac{1}{3^{s-1}}\right\}\right]\right)
$$

which numerically sums to $\frac{\lambda^{3}}{(4 \pi)^{4}} \frac{1}{\Lambda}(-2.45411725)$, and the equivalent of (12) is

$$
-24 \frac{\lambda^{3}}{(4 \pi)^{4}} \frac{1}{\Lambda}\left(\ln \frac{4}{3}+\sum_{n=1}^{\infty} \frac{(-1)^{n}}{n(n+1)}\left\{\left(\frac{2}{3}\right)^{n}-\left(\frac{1}{2}\right)^{n}\right\}\right)
$$

which equates to $12 \frac{\lambda^{3}}{(4 \pi)^{4}} \frac{1}{\Lambda}[9 \ln 3-2 \ln 2-5 \ln 5]$. The flow of the wavefunction renormalisation becomes

$$
\frac{\partial}{\partial \Lambda} Z(\Lambda)=\frac{\lambda^{2}}{(4 \pi)^{4}} \frac{1}{\Lambda} \sum_{n=2}^{\infty} \frac{(-1)^{n}}{2^{n}}=\frac{1}{6} \frac{\lambda^{2}}{(4 \pi)^{4}} \frac{1}{\Lambda} .
$$

Gathering together these equations, we obtain fast convergence to the correct $\beta$ function (14).

${ }^{a}$ Similar calculations using the exponential cutoff have been performed.8 


\section{Summary}

We have seen that convergence of the derivative expansion is an issue sensitive both to the form of cutoff and to the specific flow equation that is used. Nevertheless, the derivative expansion can converge even for massless field theory, at least to the two loops tested. If the Wilson/Polchinski flow equation is employed, convergence for the one-loop $\beta$ function can only be obtained with the use of very fast falling smooth cutoffs. Convergence at one loop is trivial if the Legendre flow equation is utilised. With a sharp cutoff in the Legendre flow equation, the expansion for the two-loop $\beta$ function also converges but clearly diverges for operators dependent on external momentum, whilst for a simple power law cutoff the expansion ceases to make sense. Finally, fast convergence of the $\beta$ function at two loops was obtained using an exponential cutoff. These results thus shed light on the accuracy seen in the non-perturbative calculations and further work along these lines could be used to bound the accuracy and reliability of practical calculations.

\section{Acknowledgements}

TRM and JFT thank PPARC for financial support.

\section{References}

1. See C. Bagnuls and C. Bervillier, hep-th/0002034 and this volume, and references therein.

2. T.R. Morris, Phys. Lett. B329 (1994) 241.

3. T.R. Morris and J.F. Tighe, JHEP 08 (1999) 007.

4. J. Polchinski, Nucl. Phys. B231 (1984), 269.

5. K. Wilson and J. Kogut, Phys. Rep 12C (1974), 75.

6. M. Bonini et al, Nucl. Phys. B483 (1997) 475.

7. T.R. Morris, Int. J. Phys. A9 (1994), 2411.

8. T. Papenbrock and C. Wetterich, Z. Phys C65 (1995), 519.

9. T.R. Morris, Nucl. Phys. B458[FS] (1996), 477. 